\title{
A NOTE ON AUTOMORPHISMS OF FINITE $p$-GROUPS
}

\author{
S. MOHSEN GHORAISHI
}

(Received 21 January 2012)

\begin{abstract}
Let $p$ be an odd prime and let $G$ be a finite $p$-group such that $x Z(G) \subseteq x^{G}$, for all $x \in G \backslash Z(G)$, where $x^{G}$ denotes the conjugacy class of $x$ in $G$. Then $G$ has a noninner automorphism of order $p$ leaving the Frattini subgroup $\Phi(G)$ elementwise fixed.
\end{abstract}

2010 Mathematics subject classification: primary 20D15; secondary 20D45.

Keywords and phrases: finite $p$-groups, automorphisms of $p$-groups, noninner automorphisms, Camina pairs.

\section{Introduction}

Let $p$ be a prime number and let $G$ be a nonabelian finite $p$-group. By a celebrated result of Gaschütz $G$ admits noninner automorphisms of $p$-power order [4]. But the existence of a noninner automorphism of order $p$ for $G$ is a long-standing conjecture for which there is as yet no counterexample [8, Problem 4.13]. The validity of the conjecture, when $G$ is a regular $p$-group, follows from a cohomological result of Schmid [9]. Abdollahi [2] has established it when $G / Z(G)$ is powerful. Deaconescu and Silberberg [3] proved that a finite $p$-group $G$ satisfying the condition $C_{G}(Z(\Phi(G))) \neq \Phi(G)$ has a noninner automorphism of order $p$ leaving the Frattini subgroup $\Phi(G)$ elementwise fixed. Liebeck [6] has shown the same result when $G$ is an odd order $p$-group of class 2. In [1], Abdollahi has shown that every 2-group of class 2 has a noninner automorphism of order two fixing $\Phi(G)$ or $\Omega_{1}(Z(G))$ elementwise.

Let $G$ be a finite $p$-group and $N$ be a nontrivial proper normal subgroup. Then $(G, N)$ is called a Camina pair if $x N \subseteq x^{G}$ for all $x \in G \backslash N$, where $x^{G}$ denotes the conjugacy class of $x$ in $G$. The main result of this paper is the following theorem.

THEOREM 1.1. Let $p$ be an odd prime and $G$ be a finite p-group such that $(G, Z(G))$ is a Camina pair. Then $G$ has a noninner automorphism of order p leaving the Frattini subgroup $\Phi(G)$ elementwise fixed.

This work was supported by the Office of Graduate Studies of the University of Isfahan, Iran.

(c) 2012 Australian Mathematical Publishing Association Inc. 0004-9727/2012 \$16.00 
Before embarking on the proof, it may be worthy of remark that Yadav has verified the divisibility conjecture for automorphisms of $p$-groups, when $(G, Z(G))$ is a Camina pair [10]. This conjecture states that for every nonabelian finite $p$-group $G$, it follows that $|G|$ divides $|\operatorname{Aut}(G)|$.

\section{Proof}

Let $G$ be a finite $p$-group. By $\mathrm{d}(G), \mathcal{M}(G)$ and $\Omega_{1}(G)$ we denote the minimum number of generators of $G$, the set of all maximal subgroups of $G$ and the subgroup of $G$ generated by all elements of order $p$, respectively. For $x \in G,\{[x, G]\}$ denotes the set $\{[x, g] \mid g \in G\}$. Any other unexplained notation is standard and follows that of Gorenstein [5].

The following lemmas are well-known results and can be verified easily.

Lemma 2.1. Let $n \in \mathbb{N}, x \in Z_{2}(G)$ and $y \in G$. Then:

(i) $\quad(x y)^{n}=x^{n} y^{n}[y, x]^{n(n-1) / 2}$;

(ii) $\left[x^{n}, y\right]=[x, y]^{n}=\left[x, y^{n}\right]$.

Lemma 2.2. Let $G$ be a finite p-group, $M$ be a maximal subgroup of $G$ and $g \in G \backslash N$. Let $u \in Z(M)$ such that $(g u)^{p}=g^{p}$. Then the map $\alpha$ given by $g \mapsto g u$ and $m \mapsto m$, for all $m \in M$, can be extended to an automorphism of $G$ and order $p$ that fixes $M$ elementwise.

Proof of Theorem 1.1. Let $(G, Z(G))$ be a Camina pair and assume that $G$ is a counterexample to the theorem.

First note that $Z(G)<Z(M)$ and $C_{G}(M)=Z(M)$, for all $M \in \mathcal{M}(G)$ [3, Remark 2].

Then we show that $Z_{2}(G)$ is abelian. It follows from [7, Theorem 2.2] that $Z_{2}(G) / Z(G)$ is elementary abelian. Therefore $x^{p} \in Z(G)$ whenever $x \in Z_{2}(G)$. Since $\Phi(G)=G^{p} G^{\prime}$, Lemma 2.1 implies that $Z_{2}(G) \leq C_{G}(\Phi(G))$. On the other hand, by the main result of [3], $C_{G}(Z(\Phi(G)))=\Phi(G)$. Therefore $C_{G}(\Phi(G))=Z(\Phi(G))$ and consequently $Z_{2}(G)$ is abelian.

Next, we claim that $|Z(G)|=p$ and $Z(M) \leq Z_{2}(G)$, for all $M \in \mathcal{M}(G)$. Let $M \in$ $\mathcal{M}(G), g \in G \backslash M$ and $x \in Z(M) \backslash Z(G)$. Since $g^{p} \in M$,

$$
\{[x, G]\}=\{[x,\langle g\rangle M]\}=\left\{\left[x, g^{i}\right] \mid 1 \leq i \leq p\right\} .
$$

Thus $\{[x, G]\}$ has at most $p$ elements. By assumption $Z(G) \subseteq\{[x, G]\}$. Therefore $Z(G)=\{[x, G]\}$ and the claim follows.

After this, we prove that $\Omega_{1}\left(Z_{2}(G)\right) \backslash Z(G) \neq \varnothing$. It follows from [10, Theorem 3.1] that $d\left(Z_{2}(G) / Z(G)\right)=d(G)$. Since $Z_{2}(G)$ is abelian,

$$
d\left(\Omega_{1}\left(Z_{2}(G)\right)\right)=d\left(Z_{2}(G)\right) \geq d\left(Z_{2}(G) / Z(G)\right) \geq 2 .
$$

Now the assertion follows because $|Z(G)|=p$.

Finally, take $u \in \Omega_{1}\left(Z_{2}(G)\right) \backslash Z(G)$ and let $M=C_{G}(u)$. Then $M \in \mathcal{M}(G)$ and if $g \in G \backslash M$, it follows from Lemma 2.1 that $(g u)^{p}=g^{p}$. By Lemma 2.2, the map $\alpha$ 
given by $g \mapsto g u$ and $m \mapsto m$, for all $m \in M$, can be extended to an automorphism of order $p$. By assumption for some $x \in G, \alpha=\theta_{x}$, the inner automorphism induced by $x$. Since $\alpha$ is the identity on $M$, we must have $x \in C_{G}(M)$ and therefore $x \in Z(M) \leq Z_{2}(G)$. This means that $u=g^{-1} g^{\alpha}=[g, x] \in Z(G)$ and contradicts our choice of $u$. The proof is complete.

\section{References}

[1] A. Abdollahi, 'Finite $p$-groups of class 2 have noninner automorphisms of order p', J. Algebra 312 (2007), 876-879.

[2] A. Abdollahi, 'Powerful $p$-groups have noninner automorphisms of order $p$ and some cohomology', J. Algebra 323 (2010), 779-789.

[3] M. Deaconescu and G. Silberberg, 'Noninner automorphisms of order $p$ of finite $p$-groups', J. Algebra 250 (2002), 283-287.

[4] W. Gaschütz, 'Nichtabelsche $p$-Gruppen besitzen äussere p-Automorphismen', J. Algebra 4 (1966), 1-2.

[5] D. Gorenstein, Finite Groups (Harper \& Row, New York, 1968).

[6] H. Liebeck, 'Outer automorphisms in nilpotent p-groups of class 2', J. Lond. Math. Soc. (2) 40 (1965), 268-275.

[7] I. D. Macdonald, 'Some p-groups of Frobenius and extra-special type', Israel J. Math. 40 (1981), 350-364.

[8] V. D. Mazurov and E. I. Khukhro (ed.), 'Unsolved problems in group theory', in: The Kourovka Notebook, No. 16 (Russian Academy of Sciences, Siberian Division, Institue of Mathematics, Novosibirisk, 2006).

[9] P. Schmid, 'A cohomological property of regular p-groups', Math. Z. 175 (1980), 1-3.

[10] M. K. Yadav, 'On automorphisms of finite p-groups', J. Group Theory 10 (2007), 859-866.

\section{S. MOHSEN GHORAISHI, Department of Mathematics, University of Isfahan, Isfahan 81746-73441, Iran \\ e-mail: m.ghoraishi@sci.ui.ac.ir}

